\title{
CORRESPONDENCE
}

\section{Role of frailty in COVID-19 patients}

\author{
Chia Siang Kow ${ }^{1 *}$ (D) and Syed Shahzad Hasan²
}

(c) 2020 Springer-Verlag GmbH Germany, part of Springer Nature

Dear Editor,

The study by Bellelli et al. [1] has suggested the importance of frailty screening upon admission with COVID19. Nevertheless, the frailty assessment tool (Frailty Index) utilized by Bellelli et al. [1] incorporated the presence/absence of various comorbidities including hypertension, cardiovascular/cerebrovascular diseases, diabetes, and chronic respiratory diseases, amongst others, as part of the assessment of frailty. In fact, the items on comorbidity constituted more than half of the items incorporated by authors for frailty assessment with the Frailty Index (23/43; 53\%). However, presence of comorbidities, especially with the aforementioned diseases, has been independently associated with worse clinical outcomes in COVID-19 patients [2]. Therefore, the true association of frailty with clinical outcomes in COVID-19 may not be illustratable with Frailty Index where comorbidity forms the majority of frailty evaluation, which could confound such association.

In this context, it is also important to re-visit the differential concepts of frailty and comorbidity. Frailty is regarded as the accumulation of subclinical declines in multiple aspects including physicality, functionality, cognition, and sociality, while comorbidity, which is regarded as the accumulation of clinically manifest diseases present in an individual, represents only one of the etiological factors of frailty [3, 4]. Therefore, frailty and comorbidity are two overlapping but distinct concepts, and both may predict disability, which is defined as a dependency in carrying out activities of daily living [4]. It is worth mentioning that Bellelli et al. also included eight items of disability, where altogether the items on comorbidity and disability constituted nearly three-quarters of

*Correspondence: chiasiang_93@hotmail.com

1 School of Postgraduate Studies, International Medical University, Kuala Lumpur, Malaysia

Full author information is available at the end of the article the total items for frailty assessment with Frailty Index (31/43; 72\%).

More useful tools for assessment of frailty in COVID19 patients, which measure frailty on multi-domain, and the presence/absence of comorbidity and/or disability do not form the majority part of the frailty evaluation, including Fried's Frailty Phenotype, Frailty Index derived from Comprehensive Geriatric Assessment, Edmonton Frailty Scale, Fatigue, Resistance, Ambulation, Illness, and Loss of Weight Index (FRAIL), Multidimensional Prognostic Index, Tilburg Frailty Index, Groningen Frailty Indicator, Sherbrooke Postal Questionnaire, and Gérontopôle Frailty Screening Tool [5] In addition, among COVID-19 patients who are severely ill and unconscious, especially those in the intensive care units who are unable to meaningfully participate in frailty assessment, Clinical Frailty Scale may be used [5]. All the aforementioned tools have a significant advantage over Frailty Index in that they capture better the multidomain phenotype of frailty, and the time required for frailty assessment is only about $5-15 \mathrm{~min}$, compared to 20-30 min typically required with Frailty Index. This is of utmost importance if we were to incorporate frailty screening as part of the triage in patients with COVID-19 since quick assessment tool is desired when dealing with overwhelming COVID-19 patients.

While we agree with authors that Frailty Index may predict outcome in COVID-19 patients, the true association of frailty with clinical outcomes in COVID19 (without much interference from comorbidities and disabilities) may be best determined with aforementioned tools, and a comparison of these suggested tools with Frailty Index is worth exploring as suggested by authors. With proper tools utilized for frailty assessment, frailty can be identified and intervened early. This is especially important among geriatric patients where frailty is prevalent to improve their clinical outcomes, shall they be hospitalized for COVID-19. 


\section{Author details}

${ }^{1}$ School of Postgraduate Studies, International Medical University, Kuala Lumpur, Malaysia. ${ }^{2}$ School of Applied Sciences, University of Huddersfield, Huddersfield, UK.

\section{Compliance with ethical standards}

\section{Conflicts of interest}

We do not have any conflicts of interest to disclose.

\section{Publisher's Note}

Springer Nature remains neutral with regard to jurisdictional claims in published maps and institutional affiliations.

Accepted: 30 June 2020

Published online: 8 July 2020

\section{References}

1. Bellelli G, Rebora P, Valsecchi MG, Bonfanti P, Citerio G (2020) COVID-19 Monza team members Frailty index predicts poor outcome in COVID-19 patients. Intensive Care Med. https://doi.org/10.1007/s00134-020-06087 $-2$

2. Guan WJ, Liang WH, Zhao Y et al (2020) Comorbidity and its impact on 1590 patients with COVID-19 in China: a nationwide analysis. Eur Respir J 55(5):2000547

3. Sacha J, Sacha M, Sobon J, Borysiuk Z, Feusette P (2017) Is it time to begin a public campaign concerning frailty and pre-frailty? Review Art Front Physiol 8:484

4. Fried LP, Ferrucci L, Darer J, Williamson JD, Anderson G (2004) Untangling the concepts of disability, frailty, and comorbidity: implications for improved targeting and care. J Gerontol Biol Sci Med Sci 59(3):255-263

5. Dent E, Kowal P, Hoogendijk EO (2016) Frailty measurement in research and clinical practice: a review. Eur J Intern Med 31:3-10 